\title{
Communication in general practice: recognition and treatment of mental illness ${ }^{1}$
}

\author{
M. Van der Pasch, P.F.M. Verhaak* \\ Netherlands Institute of Primary Care (NIVEL), P.O. Box 1568, 3500 BN Utrecht, The Netherlands
}

Received 14 November 1996; received in revised form 15 May 1997; accepted 22 June 1997

\begin{abstract}
From previous studies there is a lot of evidence that in primary care settings, many patients tend to express their mental problems in terms of physical symptoms. Therefore, the general practitioner (GP) needs to recognize mental problems at an early stage. Early recognition allows for adequate treatment that might speed up recovery. The present article reports on a study exploring the GP's ability to recognize mental illness, the communication style that is supposed to support this ability, the subsequent treatment of mental problems, and the patient's recovery. Two databases were used. First, an observation study, involving 351 videotaped consultations held by 15 GPs, yielded information on communication style and recognition abilities. Patients in this study were selected randomly. The second database obtained treatment data and measures of patient recovery from a 1-year follow-up study dealing with the treatment and course of mental illness. Patients in this study were selected because their GPs considered their problems "mainly psychosocial by nature". Half of them were categorized within psychological and social diagnostic categories of the International Classification for Primary Care (ICPC), the other half were categorized within physical disease categories, with an assessment by the GP that the complaints were mainly psychosocial. Results showed no significant relationships between the recognition of mental illness and nine communication features supposed to induce these abilities. There was a tendency however, for a positive association between recommended communicative behaviour of the GP and his or her tendency to give frequently psychosocial evaluations of the patient's complaints. Also, there was a negative tendency between this recommended behaviour and the degree of agreement between the GP's evaluation and the score on a psychiatric screening questionnaire. This agreement is called "accuracy". Frequent psychosocial evaluations were related to exploring behaviour and mental health referral in case of psychosocial complaints. Further, relationships between the GPs' recognition ability and various measures of patients' recovery did not prove univocal. Both positive, negative and absent relationships were found. (C) 1998 Elsevier Science Ireland Ltd
\end{abstract}

Keywords: Communication; General practice; Mental illness

\footnotetext{
*Corresponding author. Tel.: + 312 729700; fax: + 312729729.

${ }^{1}$ This research was partly supported by a grant from the "Nationaal Fonds Geestelijke Volksgezondheid" (National Funding for Mental Health).
} 


\section{Introduction}

In recent Dutch research it has been estimated that between 145 and 260 out of each 1000 persons who visit their general practitioner (GP) have mental problems [1]. Together these problems constitute approximately $7 \%$ of all complaints presented to the GP. The most frequent complaints are anxiety, nervousness, depression, requests for psychotropic drugs, sleep disturbances, acute stress, and problems at work.

For clarity's sake, it is critical to elucidate the terminology, used in this article, when considering mental disorder. "Mental problems", "mental distress" or "psychosocial complaints" refer to the frame of reference of the patient. It is the reason for encounter for which a patient is seeking help. Mostly all such reasons for encounter are diagnosed by a GP as "mental illness". Mental illness refers to the professional frame of reference, be it the GP or a psychiatric screening instrument. However, many more reasons for encounter, physical by nature, may be indicative for mental illness. Besides, many physical complaints may not be indicative for a mental illness, but may have important psychological consequences or backgrounds in the eye of the GP. In this case we speak of a psychosocial evaluation by the GP. A physical complaint with a psychosocial evaluation by the GP is called by us a psychosomatic complaint.

The figures cited above are rough estimates of the incidence of mental illness in general practice. Exact figures on morbidity of this kind are difficult to compile. Firstly, a substantial proportion of cases involve physical comorbidity, whereby patients present only somatic complaints [2-7]. Secondly, GPs differ significantly in the extent to which they interpret complaints as indicative for mental illness $[3,4,8,9]$.

Given the tendency of some patients to couch their problems in terms of physical symptoms, it is indeed no easy task to identify mental illness. Some GPs choose to limit their treatment to the somatic complaints presented. In contrast, GPs who suspect mental problems go beyond the information provided and give psychological treatment or refer to a mental health specialist.
Evidence of a discrepancy between a patient's "hidden" mental illness and the overt symptoms they present in general practice, as well as evidence of the assessed variability amongst GPs regarding the recognition of mental illness, is derived mainly from a line of investigation initiated by Goldberg and Huxley [8]. These investigators compared GPs' diagnoses concerning the psychosocial nature of patients' complaints to patients' scores on a screening instrument that assesses the likelihood of a person having a mental illness: the General Health Questionnaire (GHQ). Ormel et al. [10] report on 11 studies, mainly British and American, that used this paradigm to depict "hidden" (undetected) and "conspicuous" (detected) morbidity of mental illness in primary care. Frequencies of GHQ high-scorers were shown to range from $21 \%$ to $52 \%$, whereas GP cases ratings ranged between $14 \%$ to $36 \%$. Non-detection in these studies proved to be substantial; between $40 \%$ and $70 \%$ of the GP patients with an anxiety or depressive disorder were not assigned a (specific) mental diagnosis by their GP.

Differences in prevalence rates between GPs within studies have also been found to be large. In a Dutch study among 30 GPs, each interviewing about 50 patients, the proportion of complaints considered psychosocial varied between $17 \%$ and $72 \%$ [3]. In another Dutch study, carried out in eight family practices, the proportion of patients coming in for mental distress ranged from $9 \%$ to $21 \%$ across practices, with a mean of 14\% (Lamberts and Hartman, 1982 [9]. Marks et al. [11], interested in the GP's ability to accurately detect psychiatric illness, present Spearman's correlation coefficients between GHQ scores and GPs' assessments ranging between 0.09 and 0.66 .

It may not be fair to blame GPs for their inability to correctly identify all cases of GHQ. Many patients with probable mental illness visit their GP with requests for help that are of another order [12]. In fact, some investigators do not consider it desirable that GPs explicate all potential mental problems [13]. Yet it remains an interesting question, why some GPs do a better job in correctly classifying GHQ cases than other 
GPs do. It has been suggested that differences between GPs regarding their ability to correctly identify mental illness might be related to variations in their communication style. Marks et al. [11] investigated this relationship by means of an observation study and found that both verbal consultation behaviours as well as personality features differentiate the ability of GPs to detect psychiatric illness. Research psychiatrists observed 2098 interviews carried out by 55 GPs and reported on GPs' conduct in terms of a great many verbal and non-verbal classifications. GP attitudes and other personality measures were assessed as well. Recognition of mental illness was assessed by Spearman's rho between patients' GHQ scores and GPs' judgments. this measurement was labeled "accuracy". Dimensions that turned out to be significant predictors of accuracy included "interest and concern" $\left(40.5 \% \mathrm{R}^{2}\right)$, "conservatism" (negative relationship) $\left(18.5 \% \mathrm{R}^{2}\right)$, and "psychiatric focus" (5\% $\mathrm{R}^{2}$ ). Another measurement of recognition was the proportion of patients, identified by the GP as being mentally ill, without taking into account the GHQ-score. The amount of this "conspicuous morbidity", observed by the GP was predicted by GP's "interest and concern" $\left(25 \% \mathrm{R}^{2}\right)$, "psychiatric focus" $\left(16.8 \% \mathrm{R}^{2}\right)$ and "age and experience" (11\% $\left.\mathrm{R}^{2}\right)$. According to Goldberg and Huxley [8] this measurement, labelled by them as "GPs' bias", should be considered as a characteristic of the GP and not of the morbidity of his practice.

Another observation study on the relationship between consultation style and GP's recognition of mental illness was carried out by Verhaak [3]. That study did not deal with the accuracy of recognition but with the GP's bias: the tendency of the GP to interpret complaints as psychosocial. Also in this study significant relationships were reported between GPs' communication behaviours and their judgment of mental illness. GPs with a communication style characterized by bringing up many new subjects, asking many open-ended questions, showing empathy and interest, and also expressing various forms of non-verbal behaviour (nodding, agreeing) were found to be positively biased towards the inter- pretation of complaints as being psychosocial. The average consulting time was also shown to be strongly related to a bias toward psychosocial diagnosis. This communication style corresponds with the patient-centred method, advocated by Stewart et al. [14].

Goldberg et al. [15] stress the importance of such patient-centred approach, but with the addition that "it may not just be a matter of asking questions suggested by what the patient has just said but knowing when to follow up what has been said and when to move on to a new topic" (p.192).

A pivotal question, of course, is whether the recognition of mental illness matters in terms of the patient's health outcome. Some researchers $[8,10]$ have argued that non-recognition is undesirable, as it might deprive a large group of patients of appropriate treatment of their mental health problems. De Gruy [16] raises objections against this argument. In his view, recognition would not matter because, even when recognized, mental illness is treated inadequately, both in terms of dosage and duration of medications. Others again, [13], consider non-recognition less troublesome and hold that patients are quite capable of bringing up issues they want to discuss with their GP. Patients might get annoyed by a physician who keeps trying to "uncover the question behind the question".

Providing that patients can be shown to benefit from recognition and treatment of mental illnesses, we think that special training of GPs in recognizing mental illness would be an important task. Deciding on whether or not this kind of action is needed would call for knowledge of the effects of accurate recognition on subsequent treatment and on recovery. Research findings, however, do not provide definitive answers on these issues.

Ormel et al. [17] found that recognized GHQ cases as compared to GHQ cases that went unrecognized receive more mental health interventions from their GP, especially in the form of counselling and treatment with psychotropic drugs [10]. Verhaak and Wennink [18] found that an explicit demand by the patient leads to more psychological treatment; here GPs provided psy- 
chological treatment in $90 \%$ of cases when patients had explicitly stated a psychosocial reason for the encounter while in more ambiguous cases of comorbidity, GPs were found to pay no attention to the psychosocial side of the problem in $50 \%$ of the cases.

As to the effect of early recognition upon recovery, Johnstone and Goldberg [19] showed that when patients with hidden mental morbidity were identified as psychiatric cases and treated accordingly, they had a better prognosis than a control group of patients whose psychiatric illness was not detected. The detected group did better than the control group in terms of the mean duration of the illness (on average 3 versus 5 months), the total duration of psychiatric disturbance in the survey year (4 versus 6 months), and the reduction of symptoms (GHQ scores), although this last finding (GHQ scores) was only significant between subgroups with severe illness. Ormel et al. [10] showed that recognized as compared to unrecognized cases had better outcomes in terms of two screening tests, one pertaining to psychopathology and the other to social functioning. In the latter study, however, the positive effect of recognition upon recovery did not turn out to be mediated by psychological treatment. Also Verhaak and Tijhuis [7] demonstrated that treatment and recovery may be unrelated. Half of the mental health patients investigated in their study recovered after 6 months, regardless of whether they had been given treatment or not.

In sum, the literature presents conflicting conclusions. Early recognition of mental illness has been shown to be beneficial in the sense that it is often accompanied by special treatment and faster recovery. It is uncertain how this effect operates, as no one has been able to deduce recovery from the treatment.

Several accounts have been published on the relationships between communication style and early recognition of mental illness [3,4,11]. There have also been various descriptions of how early recognition of mental illness affects treatment and the course of mental illness $[7,10,17,19]$. To our knowledge, however, no one has yet investigated the overall connections between these variables. These relationships are explored in the present study. It should be seen as a preliminary exploration to obtain an overall picture on differences between GPs regarding the diagnosis and treatment of mental illness, as well as the effects on the patient's recovery. This investigation uses secondary data from a study on morbidity of mental illness carried out in the Netherlands in $1987 / 1988$ in order to gain insight into the connections between the GP's recognition ability, communication style, and treatment of mental illness and the patient's recovery. The accent is on variability between GPs.

These concerns may be expressed in the following three research questions:

1. Is there a relationship between a GP's communication style and the ability to recognize mental illness?

2. Does the ability to recognize mental illness affect the way in which these problems are treated?

3. Are differences between GPs regarding their ability to recognize mental illness related to differences in the recovery of their patients?

The answers to these questions are important for the contribution of early recognition of mental illness can to a favourable process of healing.

\section{Method}

Data for the present study were gathered within the framework of the Dutch National Study of Morbidity and Interventions in General Practice, a large-scale research project carried out in the Netherlands in 1987/1988 [20]. Those GPs who took part in this study registered each contact with every patient over a 3-month period. Each diagnosis made by the GP was assessed, which made it possible to select those patients who had been diagnosed once or more as having a mental illness. A sample of 15 GPs agreed to cooperate in two additional studies.

First, they took part in the so-called "observation" study. In this context, for each of the 15 GPs, about 25 medical interviews with random 
samples of patients were recorded on videotape. Each GP's communication skills were assessed by reviewing the videotapes, 307 in total. Also the GP's ability to recognize mental illness was inferred from this database.

Second, the 15 GPs participated in a longitudinal follow-up study dealing specifically with mental morbidity [21]. Within the frame of this follow-up study, two cohorts of patients were selected. To qualify for the study, the complaints of these patients had to have been assessed by the GP as being "mainly psychosocial" at least once. The first cohort $(N=411)$ were patients with a diagnosis classified under the chapter "P" (Psyche) or "Z" (Social) of the International Classification of Primary Care [22]. They had got at least once a diagnosis of mental illness, in the narrow sense of the word. The second cohort $(N=397)$ consisted of patients with a somatic diagnosis (all other chapters of the ICPC) that had been assessed by the GP as "mainly psychosocial in nature". These latter kind of diagnoses are labeled by us as "psychosomatic illnesses". This latter cohort did not present with any explicit psychosocial complaint during the 3 months of selection. Examples of complaints put forward by the first cohort were addiction, depression, sleepdisturbances and surmenage [21]. Of the different symptoms complained of by the patients with psychosomatic illnesses, the commonest were complaints about the locomotor apparatus, the gastrointestinal tract and the nervous system.

As patients in both cohorts presented with several reasons for visit in a number of contacts during the 3-month selection period, it is not possible to analyse homogeneous subgroups in this study, for instance only patients with depression or irritable bowel syndrome.

All contacts with the two cohorts of patients were registered for a period of 9 months after the 3 months on the National Study. During those follow-up visits, essentially the same information was recorded for these patients as had been the case during the initial 3 months of the National Study. For the selected cohorts, the follow-up study was a 9-month extension of the National Study. For each patient, the GP recorded mor- bidity and treatment details at every visit. In addition, the patients filled in a questionnaire that included the 30 item version of the General Health Questionnaire. The questionnaires were self-administered directly after the patients had been selected and again 9 months later when the follow-up registration had been completed.

The data are derived from two different studies, though they all had the 15 GPs as their common denominator. Thus, the data from the different studies had to be aggregated to the GP level. As the respondents are from different studies a multi-level approach was not possible. The next section gives an extensive overview of the main variables and their measurement.

\subsection{Recognition}

The data on the GP's recognition were derived from the "observation study". A traditional way to assess a GP's ability to recognize mental illness is to compare his/her judgment on the psychosocial nature of a patient's complaints to patients' scores on a psychiatric screening device such as the General Health Questionnaire [11]. For this purpose, the 12-item version of the GHQ was used [23]. The GHQ represents a psychiatric point of view. Respondents scoring above threshold of the GHQ have a high probability to be identified in a psychiatric clinical interview as cases of mental illness. Its sensitivity is 0.83 [24]. So, the GP's assessment has been compared with a (hypothetical) psychiatric evaluation. In line with this procedure, we assessed the agreement between the GP's psychosocial judgments and the GHQ scores of a random group of patients. The GP's assessment of the psychosocial character of a patient's complaints was based on a five-point scale, containing the categories:

- Strictly somatic

- Largely somatic

- Somatic as well as psychosocial

- Rather psychosocial

- Strictly psychosocial

Those complaints considered "strictly somatic" 
by the GP were defined as GP-minus cases. All others were defined as GP-plus cases. GHQ scores were also dichotomized. GHQ scores above the conventional cutoff point two were considered GHQ-plus cases.

Two indices of recognition, introduced by Goldberg and Huxley [8] were used. The first measure, the so-called "bias", pertains to the percentage of patients who were assigned any one of the labels "not strictly somatic". As we pointed out before, bias measures the tendency a GP has towards labelling complaints as psychosocial. No other information is used than the GP's opinion. The second recognition measure, the "accuracy", does take the GHQ as an indicator for a patient's mental illness. When using this index, the correlation is calculated between the GP's diagnosis and the assessment provided by the GHQ. The accuracy can be expressed by means of a $2 \times 2$ table, where phi $=V\left(X^{2} / N\right)$.

Table 1 presents the relationships between GHQ cases and GP cases for the entire group of GPs. Descriptors of the two recognition measures are shown in Table 2. The GP's bias in this study varies between 0.25 and 0.81 . This interdoctor variation in their tendency to make psychosocial assessments has been reported earlier by Verhaak [3]. The average bias is 0.53 . Table 1 shows that more than half of the patients were assigned a psychosocial label for any one of their complaints. This share is relatively high compared to the GHQ results, which identified 31\% of the cases as mental illness.

The accuracy figures vary between 0.00 and 0.40 with an average of 0.15 . As has been notified earlier by Marks et al. [11], there is a large

Table 1

Agreement between GP-evaluation of possible psychosocial character of complaints and score on general health questionnaire

\begin{tabular}{ccccc}
\hline & & \multicolumn{3}{c}{ General } \\
\cline { 3 - 5 } & & - & + & $n$ \\
\hline \multirow{2}{*}{ GHQ } & + & 27 & 69 & 96 \\
& - & 123 & 88 & 211 \\
$n$ & & 150 & 157 & 307 \\
\hline
\end{tabular}

inter-doctor variation in the agreement between GP's assessment and a golden (psychiatric) standard, provided by the GHQ. This indicates a variation among GPs in their ability to recognize mental illness according to psychiatric standards.

Inspection of Table 2 suggests that this low average figure must be attributed to the high rate of "false positives" - that is, the high number of GHQ-minus patients whose complaints the GP nevertheless considered (partly) psychosocial. From the other hand, $72 \%$ of the GHQ-cases have been identified by the GP as being not entirely somatic complaints. If higher cut-off points for GP's assessment were chosen, e.g. "strictly somatic" and "largely somatic" taken together as GP-minus versus the other assessments as GP-plus, the number of correct identifications decreases to $49 \%$ although the proportion of false positives decreases as well. We come back to this issue in the discussion.

\subsection{Communication style}

The "observation study" also provided the material to assess the GP's communication style. Previous studies demonstrated a link between a GP's recognition abilities and his or her communication style, the main features of which are interest, concern, verbal affective behaviour, eye contact and patient-centredness [3,11,14,25,26]. In order to reproduce these findings, a limited number of relevant communication aspects were identified by which the communication behaviours of the GPs could be characterized. This was accomplished by observation of the videotaped medical visits.

The tapes were analysed by means of the Roter Interaction Analysis System (RIAS), an instrument extensively employed in the field of patient-provider communication [26,27]. The RIAS consists of a non-verbal and a verbal part. The non-verbal part of the RIAS consists of five six-point global impression scales, by means of which observers rate a GP as to his or her overall warmth, disagreement, anxiety, and anger. The verbal part consists of 37 codes that describe each verbal expression in a medical consultation, either by patient or GP. In this study only GP 
Table 2

Descriptives of communication, recognition, treatment and recovery variables (All aggregated on GP-level, $N=15$ )

\begin{tabular}{|c|c|c|c|c|}
\hline & Mean & SD & Min & Max \\
\hline \multicolumn{5}{|l|}{ Communication: } \\
\hline \multicolumn{5}{|l|}{ Affective behaviour } \\
\hline Attentiveness & 0.04 & 0.34 & -0.60 & 0.47 \\
\hline Disagreement & -0.03 & 0.31 & -0.52 & 0.63 \\
\hline Showing concern & 0.06 & 0.53 & -1.16 & 0.93 \\
\hline Social behaviour & 0.03 & 0.33 & -0.39 & 0.57 \\
\hline \multicolumn{5}{|c|}{ Instrumental behaviour } \\
\hline Questions & 4.2 & 2.8 & 1.6 & 12.2 \\
\hline Information & 2.4 & 2.0 & 0.2 & 8.8 \\
\hline Counseling/advice & 2.3 & 1.8 & 0.7 & 7.1 \\
\hline \multicolumn{5}{|l|}{ Influence for patient } \\
\hline In diagnostic stage & 3.01 & 0.42 & 2.0 & 3.6 \\
\hline In therapeutic stage & 3.10 & 0.31 & 2.6 & 3.7 \\
\hline \multicolumn{5}{|l|}{ Recognition } \\
\hline Bias & 0.53 & 0.20 & 0.25 & 0.81 \\
\hline Accuracy & 0.15 & 0.12 & 0.03 & 0.40 \\
\hline
\end{tabular}

\section{Treatment}

Treatment of psychosocial complaints

Explicitation

Listening/reassur.

14

27.5

Exploring

Information

Await further devel.

Psychoactive drugs

Referral mental health

Consultation ment. hlt.

Consultation $>10 \mathrm{~min}$

Treatment of psychosomatic complaints

Explicitation

$40 \%$

$48 \%$

$50 \%$

$27 \%$

$3 \%$

$11 \%$

$4 \%$

$3 \%$

$54 \%$

Listening/reassur.

Exploring

Information

Await further developm.

Psychoactive drugs

Referral mental health

Consultation ment. hlt.

Consultation $>10 \mathrm{~min}$.

Treatment of somatic complaints

Explicitation

Listening/reassur.

Exploring

Information

$34 \%$

$22 \%$

$28 \%$

$48 \%$

$3 \%$

$3 \%$

$1 \%$

$0 \%$

$36 \%$

Await further developm.

Psychoactive drugs

Referral mental health

Consultation ment. hlt.

Consultation $>10 \mathrm{~min}$.
24.5

20.8

3.1

10.5

2.3

3

21.5

11.8

15.4

19.4

26.6

2.4

3.8

0.7

0.4

23.7

5.2

4.2

10.3

27.7

1.9

1.2

$-$

0.2
$10 \%$

$6 \%$

$8 \%$

$2 \%$

$0 \%$

$0 \%$

$1 \%$

$0 \%$

$13 \%$

$14 \%$

$3 \%$

$4 \%$

$9 \%$

$0 \%$

$0 \%$

$0 \%$

$0 \%$

$4 \%$

$62 \%$

$86 \%$

$88 \%$

$70 \%$

$9 \%$

$30 \%$

$10 \%$

$9 \%$

$86 \%$

$60 \%$

$54 \%$

$66 \%$

$83 \%$

$9 \%$

$15 \%$

$2 \%$

$1 \%$

$76 \%$

$\begin{array}{ll}1 \% & 21 \% \\ 0 \% & 16 \%\end{array}$

$\begin{array}{ll}0 \% & 16 \% \\ 0 \% & 31 \%\end{array}$

$6 \% \quad 89 \%$

$0 \% \quad 6 \%$

$0 \% \quad 3 \%$

$-$

$0 \%-1 \%$ 
Table 2. Continued

\begin{tabular}{|c|c|c|c|c|}
\hline & Mean & $\mathrm{SD}$ & Min & Max \\
\hline \multicolumn{5}{|l|}{ Communication: } \\
\hline \multicolumn{5}{|l|}{ Recovery after 12 months } \\
\hline \multicolumn{5}{|l|}{ Cohort with explicit psychosocial complaints } \\
\hline Decrease in GHQ-30 & 1.7 & 1.8 & -1.1 & 6.8 \\
\hline Decrease in psychosoc. complaints $/ 3$ months & 1.3 & 0.3 & 0.9 & 1.8 \\
\hline Decrease in psychosom. complaints $/ 3$ months & 0.3 & 0.3 & -0.2 & 0.9 \\
\hline Decrease in somatic complaints $/ 3$ months & 0.3 & 0.2 & -0.1 & 0.5 \\
\hline \multicolumn{5}{|l|}{ Cohort with psychomatic complaints } \\
\hline Decrease in GHQ-30 & 0.1 & 2.1 & -3.5 & 3.8 \\
\hline Decrease in psychosoc. complaints $/ 3$ months & -0.1 & 0.1 & -0.3 & 0 \\
\hline Decrease in psychosom. complaints $/ 3$ months & 0.4 & 0.5 & -0.3 & 1.6 \\
\hline Decrease in somatic complaints $/ 3$ months & 1.1 & 0.1 & 0.9 & 1.4 \\
\hline
\end{tabular}

data are analysed as GP's behaviour is the main focus of interest.

The verbal part of the RIAS consists of two main groups of codes. The affect group has 12 codes reserved for socio-emotional exchange, such as worry, concern, agreement etc. The task group contains 25 codes, pertaining to task-focused exchange; this refers to questions, giving information, and counselling on somatic or psycho-emotional topics. (The entire system is shown in the Appendix). Here, only the task categories pertaining to psycho-emotional topics are taken into consideration. The RIAS was coded directly from the videotape. The total scores for the verbal part were calculated by tallying the number of remarks made in each category.

In addition to the RIAS, two other communication aspects were assessed for which the relevance to recognition has been shown in the literature: patient's influence in assessment and decision making and eye contact. The concept of patient's influence was developed by Byrne and Long [28]. It refers to the degree in which a GP takes into account the patient's views and ideas about illness and treatment [29]. Patient's influences was assessed separately for the diagnostic and the treatment phases of the consultation by means of a five-point scale. A GP's eye contact is a form of nonverbal communication that is considered important in two ways. On the one hand, eye contact provides the GP with information on a patient's emotional state. On the other hand, it is an important means to convey interest and empathy, features that are considered necessary for making patients feel safe enough to talk straightforwardly about their worries [25]. The eye contact in this study was specified as the length of time the GP looked directly into the patient's face. It was measured by stopwatch.

Condensing the affective RIAS measures was accomplished by factor analysis of the scores of the 12 affective codes, the 5 global impression ratings, and the eye contact variable. A principal components analysis revealed four factors: attention, interest/concern, disagreement, and social behaviour. Table 3 depicts the factor items and their loadings. These four factors, the GP's total scores on three task categories pertaining to psychological anamnesis, and the two global impression ratings on the influence the patient has got, formed the final set of variables of communication style. To be able to relate these communication variables to a GP's recognition ability, all variables were aggregated to the GP level. Table 2 gives an overview of the frequencies of the communication style indicators. The figures are presented at the aggregated GP level $(n=15)$.

This extended RIAS-schedule proved to be reliable: 20 consultations observed by three separate observers yielded Pearson's product-moment correlations between 0.72 and 0.91 for the affective clusters, between 0.77 and 0.94 for the instrumental clusters, between 0.50 and 0.89 for 
Table 3

Factor-structure of affect-variables

\begin{tabular}{lcccc}
\hline Factor-items & Factor 1 attention & Factor 2 disagreement & Factor 3 interest/concern & Factor 4 social behaviour \\
\hline Agreement & 0.78 & 0.08 & 0.09 & 0.16 \\
Paraphrases & 0.76 & 0.06 & 0.13 & -0.01 \\
Eye contact & 0.73 & 0.05 & 0.15 & 0.30 \\
Legitimation & 0.68 & 0.26 & 0.18 & -0.00 \\
Empathy & 0.62 & -0.13 & -0.04 & -0.04 \\
Anger (global) & 0.01 & 0.79 & -0.01 & 0.21 \\
Disagreement & 0.04 & 0.76 & 0.19 & -0.06 \\
Anxiety (glb) & 0.15 & 0.65 & -0.22 & 0.02 \\
& & & & 0.02 \\
Dominance(glb) & -0.21 & -0.01 & 0.69 & 0.13 \\
Interest (glb) & 0.28 & -0.38 & 0.69 & 0.01 \\
Warmth (glb) & 0.28 & -0.43 & 0.68 & 0.30 \\
Worry & 0.22 & 0.30 & 0.40 & 0.30 \\
Partnership & 0.24 & 0.12 & 0.39 & 0.70 \\
Reassurance & 0.30 & 0.12 & -0.05 & 0.54 \\
Personal remarks & 0.11 & -0.01 & 0.02 & 0.50 \\
Approval & 0.01 & -0.06 & 0.17 & \\
Jokes & 0.14 & 0.13 &
\end{tabular}

the global ratings and 0.97 or higher for the non-verbal measurements [30]

\subsection{Treatment}

Treatment data were gathered from the registration study. In the course of 1 year, 808 patients put forward 5815 reasons for an encounter (complaints). About $20 \%$ of these reasons were of psychosocial, $30 \%$ of psychosomatic and $50 \%$ of a purely somatic nature. All encounters as well as their treatment were recorded by the GP on a special form. The following eight variables were derived from those records: making explicit psychosocial suspicions, passive counselling: listening and reassurance, active counselling: exploring, providing information, await further developments, prescription of psychotropic drugs, referrals to mental health specialists or institutions, consultation of mental health specialists, duration of consultation.

As these data should be analysed on GP level, they were aggregated on GP-level, separately for psychosocial reasons for encounter, psychosomatic reasons for encounter and purely somatic reasons for encounter. This results in measurements on GP-level like "proportion of consultations with psychosocial reasons for encounter where the GP prescribed psychotropic drugs" or "proportion of consultations with psychosomatic complaints where the GP made explicit his suspicion of mental disorder".

Table 2 presents descriptors of the treatment variables at the aggregate level.

In case of psychosocial complaints, the average GP counsels a lot: active as well as passive counselling is registered in about half of the cases. In $40 \%$ the psychosocial character of the complaints is discussed explicitly. More than half of the consultations last longer than 10 minutes. There are large differences between GPs: some register these "psychotherapeutic" indicators almost always in case of psychosocial complaints, some do it hardly at all. Psychofarmacological drugs are prescribed in about $11 \%$ of the cases. As only prescription by the GP himself during the consultation is taken into account (not counting repeat prescriptions, handled by the practice assistant), this is an underestimation of the real psychofarmacological treatment in general practice [6]. A referral or consultation of mental health specialist is a rare observation. 
All these treatment activities decrease, when we look at psychosomatic and especially at somatic complaints, except for information giving, which in turn increases. However, in case of psychosomatic complaints a large inter-doctor variation remains for the "psychotherapeutic" indicators.

\subsection{Recovery}

Indicators for recovery are deduced from a comparison between the first 3 months of the registration data and the last 3 months. Two kinds of indicators of recovery were used [21]. In the first place, the difference between the patients' initial and final GHQ scores was assessed. That is, the scores recorded at the beginning of the registration study were compared with the patients' GHQ scores 9 months later, at the end of follow-up period.

The second indicator of recovery was based on the number of times a patient had visited the GP during the year of investigation with either psychosomatic or psychosocial complaints. The difference between the medical consumption in the first and fourth period of the study was the second recovery index. Table 4 shows the descriptors for each of these measures.

For both groups of patients, the number of psychosocial and somatic complaints, presented during 3 months decreased significantly. Patients who were selected with explicit psychosocial complaints had a significantly lower GHQ-score and reported less psychosocial complaints after 1 year, while the GHQ-score of patients selected because of their psychosomatic complaints remained at the same level. These latter patients did not present explicit psychosocial complaints during the 3 months of selection (this was an exclusion-criterium), so they had a slight increase in psychosocial complaints.

\section{Results}

\subsection{Recognition and communication style}

The first main question is whether or not differences among GPs regarding the recognition of mental illness coincide with differences in their style of communication.

To examine the relationships between the variables of communication style and the GP recognition indices of "bias" and "accuracy", a correlation analysis was carried out at the GP level $(n=15)$. The results of this analysis are presented in Table 4. These correlations do not reach the level of statistical significance due to the small $N$. Nevertheless, the negative trend between nearly all communication variables and the accuracy is striking, as opposed to the neutral or positive trend between communication variables and the bias of the GP in a psychosocial direction. The lack of statistically significant

Table 4

Correlation coefficients between communication and recognition variables $(N=15)$

\begin{tabular}{lrc}
\hline Communication variables & Bias & Accuracy \\
\hline Affective behaviour & & -0.24 \\
Attentiveness & 0.29 & -0.09 \\
Showing concern & 0.23 & -0.42 \\
Social behaviour & 0.40 & 0.14 \\
Disagreement & & -0.56 \\
Instrumental behaviour & 0.38 & -0.26 \\
Questions on psycho-emotional subjects & -0.01 & -0.05 \\
Information on psycho-emotional subjects & 0.03 & -0.43 \\
Counseling and advice on psycho-emotional subjects & & -0.14 \\
Global ratings & 0.17 & 0.14 \\
Patient-influence in diagnostic phase & & \\
Patient-centeredness in therapeutic phase & & \\
\hline
\end{tabular}


relationships is due to the small number of cases, as many of the correlation figures are actually quite substantial.

The positive trend between the communication variables and the bias was stronger on the level of the individual consultation (Table 5). In consultations were the GP suspected a mental illness, there was more verbal attention, social behaviour, instrumental psychosocial behaviour, influence for the patient and interest and concern. However, there was hardly any relationship between this "patient-centred behaviour" and the agreement between the GP's evaluation and the GHQ-score in the consultation concerned (i.e. the accuracy). This kind of behaviour does not seem a necessary prerequisite for a judgment that matches the results of a psychiatric screening instrument.

\subsection{Recognition and treatment}

The second question concerns the relationship between recognition and treatment. Does the ability to recognize mental illness affect the way in which these problems are treated?

We carried out a correlation analysis between the two recognition indices, bias and accuracy, on the one hand and the various treatment indicators on the other. The correlation matrix is depicted in Table 6 . It shows that the bias, the
Table 6

Correlation coefficients between treatment and recognition variables (GP-level, $N=15)$

\begin{tabular}{lcc}
\hline & Bias & Accuracy \\
\hline Treatment of psychosocial complaints & \\
Explicitation & -0.13 & 0.14 \\
Listening/reassur. & 0.06 & 0.35 \\
Exploring & $0.72 * *$ & 0.10 \\
Information & -0.03 & 0.30 \\
Await further devel. & -0.37 & -0.07 \\
Psychoactive drugs & -0.05 & 0.19 \\
Referral mental health & $0.54^{*}$ & -0.04 \\
Consultation ment. hlt. & 0.35 & -0.31 \\
Consultation $>10$ min. & 0.38 & -0.31 \\
& & \\
Treatment of psychosomatic complaints & \\
Explicitation & 0.06 & 0.08 \\
Listening/reassur. & 0.06 & 0.09 \\
Exploring & 0.39 & 0.23 \\
Information & 0.24 & 0.37 \\
Await further developm. & -0.15 & -0.23 \\
Psychoactive drugs & 0.29 & -0.13 \\
Referral mental health & -0.20 & -0.19 \\
Consultation ment. hlt. & 0.26 & 0.14 \\
Consultation $>10$ min. & 0.43 & 0.29 \\
\hline
\end{tabular}

* $P<0.01$.

** $P<0.05$.

extent to which GPs tend to consider complaints psychosocial, is related to active counselling and referring to mental health in case of psychosocial complaints. There is a trend for longer consultations in these cases. Accuracy does not affect the

Table 5

Correlation between communication indices and identification indices on consultation level $(N=307)$

\begin{tabular}{llr}
\hline Communication indices: & Bias & Accur. \\
\hline Affective behaviour: & & $0.32^{* *}$ \\
Verbal attentiveness & $0.10^{*}$ & 0.05 \\
Showing concern & $0.15^{* *}$ & 0.01 \\
Social behaviour & 0.08 & $-0.15^{*}$ \\
Disagreement & & -0.02 \\
Instrumental/task related: & $0.28^{* *}$ & $-0.19^{* *}$ \\
Questions on psycho-emotional subjects & $0.23^{* *}$ & -0.06 \\
Information on psycho-emotional subjects & & -0.04 \\
Counseling/advices on psycho-emotional subjects & & -0.06 \\
Patient centredness & $0.29^{* *}$ & -0.07 \\
Diagnosis & $0.26^{* *}$ & \\
Treatment & & \\
\hline
\end{tabular}

$* P<0.05$.

** $P<0.01$. 
way GPs treat psychosocial problems. There is a trend to give more information in case of psychosomatic problems, physical complaints assessed by the GP as probably psychosocial by nature. When all kinds of reasons for encounter are taken together, those who make a more accurate diagnosis prescribe more psychotropic drugs and provide more information. The accent on information might be a consequence of the type of drugs prescribed. This is because explaining dosages, informing the patient about possible side-effects, and stressing the importance of continuing with the drugs are all classified as "information giving".

\subsection{Recognition and recovery}

As said earlier, three indicators of patients' recovery were assessed: patients' recovery as indicated by the decline of patients' GHQ score, and patients' recovery in terms of the decline in number of psychosocial complaints and psychosomatic complaints. In order to explore whether GPs recognition ability was related to their patients' faster recovery, GPs scoring high and low regarding bias and accuracy were compared on these three recovery measures. The recovery measures of patients seen by the 5 GPs scoring highest on recognition were compared with the recovery measures of patients seen by the patients scoring lowest.

Table 7 shows the results. The GHQ-difference scores do not seem influenced by the GPrecognition measures, be it "bias" or "accuracy". Recovery in terms of decrease in number of complains, however, does seem connected to the GPs' recognition ability. Patients of the GPs with upper scores on "bias" present a stronger decline of psychosocial complaints than their colleagues with lower "bias" scores. As for the recovery of psychosomatic complaints it was found that, surprisingly, more progress for this type of problems was assessed with GPs low in "accurate" recognition. This as opposed to high accurate GPs.

\section{Discussion}

This study investigated differences between GPs regarding their recognition abilities, the ways in which they communicate with patients, their treatment habits, and the effect of these behaviours upon their patients' recovery.

Two recognition indices were assessed: the GP's tendency to take psychosocial aspects into consideration, the "bias"; and agreement between assessments made by different GPs and a psychiatric screening questionnaire, their "accuracy". We related these recognition measures to communication aspects that supposedly give rise to early recognition. A positive trend showed up between the communication variables and the GP's bias as opposed to a negative trend between communication and the GP's accuracy. As for treatment, highly biased GPs demonstrated more exploring behaviour and mental health referral in case of psychosocial complaints. Taken all complaints together, accurate GPs prescribed more psychopharmacological drugs than less-accurate GPs. Recovery of patients of highly biased GPs, though, was more favourable than recovery of patients of less-biased GPs; the former groups showed a larger decrease in psychological complaints. Patients of GPs scoring high on accuracy, on the other hand, showed less

Table 7

Comparison of three measures of recovery of patients of the five highest $(+)$ and the five lowest $(-)$ GPs concerning recognition

\begin{tabular}{|c|c|c|c|c|c|c|c|c|c|}
\hline \multirow[b]{2}{*}{ I Recognition variables low versus high scores } & \multicolumn{3}{|c|}{ GHQ1-GHQ2 } & \multicolumn{3}{|c|}{$\begin{array}{l}\text { Psychosocial com- } \\
\text { plaints } 1-2\end{array}$} & \multicolumn{3}{|c|}{$\begin{array}{l}\text { Psychosomatic com- } \\
\text { plaints } 1-2\end{array}$} \\
\hline & - & + & $T$ & - & + & $T$ & - & + & $T$ \\
\hline Bias & 1.31 & 0.24 & 1.6 & 0.29 & 0.60 & $-2.8^{*}$ & 0.52 & 0.57 & -0.7 \\
\hline Accuracy & 1.20 & 0.92 & 0.5 & 0.39 & 0.46 & -0.7 & 0.61 & 0.48 & $1.8^{* *}$ \\
\hline
\end{tabular}

$* P<0.05$.

** $P<0.10$. 
recovery, that is less decline in psychosomatic complaints, than patients of less-accurate GPs.

Some findings of this study warrant further discussion. An atypical finding of this study concerns the rather high rate of mental illness identified by the GPs. In $51 \%$ of all cases, GPs considered the complaints to be at least partly psychosocial in nature. In a previous study among 30 GPs (1500 consultations) 10 years ago, using a comparable method for assessing GP's bias we found similar figures [3]. These figures are high compared to the amount of mental illness $(31 \%)$ detected by the GHQ. The figure of $51 \%$ is also high compared to the mental morbidity ratings mentioned in a review by Ormel et al. [10], where conspicuous morbidity figures range from $14 \%$ to $36 \%$. On the basis of these findings, under-recognition does not appear to be a problem. However, many previous recognition studies compared GHQ scores with the GPs' opinions of the general mental state of their patients. In the present study, GPs were asked to assess the psychosocial nature of their patients complaints. Moreover, the present study uses a low threshold for the GP's assessment: complaints assessed as "largely somatic" (2) or "somatic as well as psychosocial" (3) are counted as a psychosocial assessment by the GP. An increase of this threshold substantially reduces the number of psychosocial assessments and the proportion of identified GHQ-cases as well.

We had not expected the negative trend that showed up between the communication variables and the accuracy of the GP's judgment on the psychosocial nature of patients' complaints. This finding is puzzling. In earlier studies, communication aspects such as interest, empathy, patient centredness, and eye contact were shown to be facilitating conditions. These behaviours stimulated patients to talk about themselves. The puzzle might be solved by differentiating patients whose problems should be considered as having a psychological dimension - without being classified as mental illness, in terms of the GHQ from patients who are mentally ill in a narrow sense. We shall return to this issue later

With respect to the figures on recovery, the findings seem somewhat puzzling too. Hardly any association was found between bias and treatment. Nonetheless, patients seen by GPs who scored high on bias had better recovery results than patients of GPs who scored low on bias. Patients of GPs who scored high on accuracy, on the other hand, showed less recovery than patients of GPs who scored low on accuracy. This paradoxical result may be a consequence of the bias/accuracy problem, which will be discussed below. Besides, relationships between recognition, treatment, and recovery should be interpreted carefully. The amount of treatment a GP provides may depend on early recognition. But it may also depend on the severity of illness, the wishes and expectancies of the patient and the appropriateness of treatment, neither of which were measured in this study. As Ormel et al. [10] pointed out, the less effective GPs are in their interventions with a particular patient, the greater the range of treatment they will provide. Consequently, correlations between treatment and recovery may be low, absent, or even inverse.

An important issue that emerged in this study is the possible difference between patients whose complaints are not merely physical, though they are not psychiatric cases, and patients who might be psychiatrically ill. When we take the agreement between GHQ (screening for the psychiatrically ill) and the GP's assessment (covering all patients who are not merely physically ill) into account, as shown in Table 1, the former group seems to be a subset of the latter. Let us call the former group the psychiatric cases and the latter the psychosocially disturbed patients. A substantial share of those who are psychosocially disturbed according to the GP are not psychiatric cases according to the GHQ; hence, they are "false positives". However, they are "false positives" from a psychiatric point of view, as we should keep in mind that the GHQ is an indicator for a psychiatrist's clinical judgment. From a general practice point of view, these patients too deserve care and attention from their GP, because a GP takes care for the person as a whole, including his worries and fears [14]. The recognition measurement "bias" acknowledges this task of the GP. The measurement 
"accuracy", however, punishes the GP for paying attention to patients who are merely psychosocially disturbed without being a case of psychiatric illness. GPs who appear to be the most accurate ones neglect a substantial part of the minor psychosocial problems, the anxieties and worries that accompany the visit to a doctor. Perhaps they are sensitive in a technical sense, but they might be deficient from the human angle, as indicated by our measures of attentiveness and social behaviour. Indeed, they are often in conflict with the patient, as indicated by the reported disagreements.

This interpretation also corresponds to the finding that as far as treatment is concerned, the "accurate" GP distinguishes him or herself by the tendency to prescribe psychoactive drugs. This interpretation is also in accordance with the finding that GPs who interpret many complaints as being not merely physical see a greater reduction in psychosocial complaints among their patients after a year than GPs who interpret few complaints as having a psychological dimension. A high or low degree of accuracy hardly matters in this respect.

In summary, recognition of mental illness is a complicated matter to study. If one focuses on the GP's accuracy in selecting those patients with a mental disorder in the narrow sense, one does not acknowledge the task specific to family practice. An "accurate" doctor detects relatively many mentally disturbed patients, but he should also recognize emotional disturbance in mentally healthy patients too. It is an ominous sign that high accuracy is associated with non-affective and doctor-centred communicative behaviour. Although most of the correlation coefficients are not statistically significant, they all point in a negative direction. On the other hand, the "psychologically biased" GP does communicate in the recommended way. As far as any beneficial results could be detected, these were found among the patients of the most highly biased doctors. Non-detection of psychiatric illness must be avoided in all cases. However, attention to emotional disturbances that are not psychiatric illnesses in the narrow sense is equally important.

This investigation has some obvious limitations. Perhaps the most fundamental one is that we used diverse sets of secondary data, not specifically gathered for purpose of this study. One of the problems this engendered is the unequal size of the data sets. Because the sizes differed, we had to analyse our data on an aggregated GP level. That, in turn, caused loss of information and made it difficult to obtain significant results. Another problem stemming from the research design is that the registration study only included patients whose mental health was assessed by the GP. In effect, patients with mental illness that were not detected by the GP were not included. This may have caused a restriction of range effect. If random groups of patients would have participated, larger ranges of treatment might have been found. In the same vein, the correlations between recognition and treatment would undoubtedly have been stronger, especially among patients with psychosomatic morbidity. Another methodological problem, related to the ones already mentioned, is the heterogenity of the groups of included patients.

One last methodological flaw concerns the absence of variables that are highly relevant to the course of mental illness. In the model investigated, only GP variables were included. Obviously, patient variables such as severity of illness, onset of illness, etc. may play an equally important role. They may even confound or modify the relationship between recognition, treatment, and recovery.

To give more nuance to these coarse findings, additional studies are necessary. Further studies should include a larger group of GPs, use a more refined design, and take relevant patient characteristics into account as well.

\section{Appendix 1}

\section{Roter's interaction analysis system (RIAS)}

\section{Socioemotional exchange}

1. Personal remarks, social conversation.

2. Laughing, jokes. 
3. Gives compliments, shows approval.

4. Shows agreement or understanding.

5. Paraphrases/interprets.

6. Empathy.

7. Shows concern or worry.

8. Reassures, encourages or shows optimism.

9. Legitimizes.

10. Shows partnership and support.

11. Show disagreement, misunderstanding or criticism.

12. Asks for reassurance.

\section{Task-focused exchange}

13. Transition words.

14. Gives orientation, continuity prompts or instructions.

15. Asks for clarification.

16. Asks for understanding or confirmation of understanding.

17. Asks for opinion.

18. Asks questions (closed-ended) - medical condition.

19. Asks questions (closed-ended) - therapeutic regimen.

20. Asks questions (closed-ended) - lifestyle.

21. Asks questions (closed-ended) - feelings and emotions.

22. Asks questions (closed-ended) - other.

23. Asks questions (open-ended) - medical condition.

24. Asks questions (open-ended) - therapeutic regimen.

25. Asks questions (open-ended) - lifestyle.

26. Asks questions (open-ended) - feelings and emotions.

27. Asks questions (open-ended) - other.

28. Gives information - medical condition.

29. Gives information - therapeutic condition.

30. Gives information - lifestyle.

31. Gives information - feelings and emotions.

32. Gives information - other.

33. Counsels or directs behaviour - medical condition/therapeutic regimen.

34. Counsels or directs behaviour - lifestyle.

35. Counsels feelings.

36. Request for medications.

37. Unintelligible.

\section{References}

[1] Verhaak P, Bijl RV, van de Bergh-Jeths A, Harteloh PPM. Psychische problema tiek. [Psychological problems] In: Ruwaard D, Kramers, PGN, editors. Volksgezondheid Toekomstverkenning RIVM. Den Haag: SDU, 1993.

[2] Goldberg DP, Blackwell B. Psychiatric illness in general practice, a detailed study using a new method of case identification. Br Med J 1970;2:439-43.

[3] Verhaak PFM. Interpretatie en behandeling van psychosociale klachten in de huisartspraktijk. [Interpretation and treatment of psychosocial complaints in general practice] Utrecht. Thesis, 1986.

[4] Verhaak PFM. Variations in the diagnosis of psychosocial disorders: a general practice observation study. Soc Sci Med 1986;23:595-604.

[5] Verhaak PFM. Detection of psychological complaints by general practitioners. Med Care 1988;26:1009-20.

[6] Verhaak PFM. Mental disorder in the community and in general practice. Aldershot: Avebury, 1995.

[7] Verhaak PFM, Tijhuis MAR. Psychosocial problems in primary care: some results from the Dutch national study of morbidity and interventions in general practice. Soc Sci Med 1992;35:105-10.

[8] Goldberg D, Huxley P. Mental Illness in the Community. The pathway to Psychiatric Care. London: Tavistock, 1980.

[9] Lamberts H, Hartman B. Psychische en sociale problemen in de huisartspraktijk. [Psychological and Social Problems in General Practice]. Huisarts Wet 1982;25:333-42.

[10] Ormel J, Koeter MWJ, van den Brink W, van de Willige G. The extent of non-recognition of mental health problems in primary care and its effects on management and outcome. In: Goldberg D, Tantam D, editors. The Public Health Impact of Mental Disorder, 1990.

[11] Marks JN, Goldberg DP, Hillier VF. Determinants of the ability of general practitioners to detect psychiatric illness. Psychol Med 1979;9:337-53.

[12] Bensing J, Beerendonk PM. Psychosociale problemen in de huisartspraktijk: weten en meten. [Psychosocial problems in general practice: knowing and measuring]. Maandblad Geestelijke Volksgezondheid 1990;45:595618.

[13] Hofmans-Okkes IM. Op het spreekuur. Oordelen van patiënten over huisartsconsulten. [In the consultationroom. Patients evaluating their GP] Lelystad: Meditekst. University of Amsterdam. Thesis, 1991.

[14] Stewart M, Brown JB, Weston WW, McWhinney IR, McWilliam CL, Freeman TR. Patient centred medicine. Transforming the clinical method. Thousand Oaks: Sage Publications, 1995.

[15] Goldberg DP, Jenkins L, Millar T, Faragher EB. The ability of trainee general practitioners to identify psychological distress among their patients. Psychol Med 1993;23(1):185-193.

[16] deGruy F. Mental health care in the primary care 
setting. In: Donaldson MS, Yordi KD, Lohr KN, Vanselow NA, editors. Primary care. America's health in a new era. Washington: National Academy Press, 1996:285-311.

[17] Ormel J, Koeter MWJ, Giel R, van der Meer K, van de Willige G, Wilmink FW. Recognition, management and outcome of psychological disorders in primary care: a naturalistic follow-up study. Psychol Med 1990;20:90923.

[18] Verhaak PFM, Wennink HJ. What does a doctor do with psychosocial problems in primary care?. Int J Psychiatry Med 1990;20:151-62.

[19] Johnstone A, Goldberg D. Psychiatric screening in general practice. A controlled trial. Lancet 1976;20:6058.

[20] Foets M, and van der Velden J. Nationale studie naar ziekten en verrichtingen in de huisartspraktijk. Basisrapport Meetinstrumenten en procedures [Dutch national study on morbidity and interventions in general practice. Measuring instruments and procedures]. Utrecht: Nivel, 1990.

[21] Tijhuis MAR, Verhaak PFM, Wennink HJ, Psychosociale Problemen in de Huisartspraktijk. Een Nationale Studie van Ziekten en Verrichtingen in de Huisartspraktijk [[Dutch national study on morbidity and interventions in general practice. Psychological Problems]. Utrecht: Nivel, 1991.

[22] Lamberts $H$, Wood $M$, editors. ICPC. International classification for primary care. Oxford: Oxford University Press, 1987.

[23] Goldberg DP, The detection of psychiatric illness by questionnaire. London: Oxford University Press, 1972.

[24] Goldberg D. Identifying psychiatric illness among general medical patients. Br Med J 1985;291:161-2.

[25] Bensing JM, Kerssens JJ, van der Pasch, MAA. Patientdirected gaze as a tool for discovering and handling psychosocial problems in general practice. J Nonverbal Behav, 19:223-241.

[26] Bensing J. Doctor-patient communication and the quality of care. Utrecht: Nivel. Thesis, 1991.

[27] Roter D. The Roter method of interaction process analysis. Baltimore: Johns Hopkins University (unpublished manuscript), 1989.

[28] Byrne PS, Long BEL. Doctors talking to patients. London: Her Majesty's Stationary Office, 1976.

[29] Stewart MA. What is a successful doctor-patient interview? A study of interactions and outcomes. Soc Sci Med 1984;19:167-75.

[30] Brink-Muinen Avd. Gender, health and health care in general practice. Utrecht: Nivel, Thesis, 1996. 